\title{
BMJ Open Trabeculotomy versus combined trabeculotomy-trabeculectomy for primary congenital glaucoma: study protocol of a randomised controlled trial
}

\author{
Lei Fang, ${ }^{1}$ Xinxing Guo, ${ }^{1,2}$ Yangfan Yang, ${ }^{1}$ Jian Zhang, ${ }^{1}$ Xiangxi Chen, ${ }^{1,3}$ \\ Yingting Zhu, ${ }^{1}$ Jielei Huang, ${ }^{1,4}$ Jingjing Huang, ${ }^{1}$ Yimin Zhong, ${ }^{1}$ Xiaoyu Xu, ${ }^{1}$ \\ Xing Liu (1) ${ }^{1}$
}

To cite: Fang L, Guo X, Yang Y, et al. Trabeculotomy versus combined trabeculotomytrabeculectomy for primary congenital glaucoma: study protocol of a randomised controlled trial. BMJ Open 2020;10:e032957. doi:10.1136/ bmjopen-2019-032957

- Prepublication history and additional material for this paper are available online. To view these files, please visit the journal online (http://dx.doi. org/10.1136/bmjopen-2019032957).

Received 14 July 2019 Revised 21 January 2020 Accepted 05 February 2020

D) Check for updates

(c) Author(s) (or their employer(s)) 2020. Re-use permitted under CC BY-NC. No commercial re-use. See rights and permissions. Published by BMJ.

${ }^{1}$ State Key Laboratory of Ophthalmology, Zhongshan Ophthalmic Center, Sun Yat-sen University, Guangzhou, China

${ }^{2}$ Wilmer Eye Institute, Johns Hopkins University, Baltimore, Maryland, USA

${ }^{3}$ Department of Cornea, Wuhan Aier Eye Hospital, Wuhan, China ${ }^{4}$ Department of optometry, Zhongshan Aier Eye Hospital, Zhongshan, China

Correspondence to

Dr Xing Liu; drliuxing@163.com

\section{ABSTRACT}

Introduction Trabeculotomy and combined trabeculotomytrabeculectomy (CTT) are major surgical options for primary congenital glaucoma (PCG). However, it is unclear which of these two surgical procedures should be recommended as the optimum first-line treatment for PCG. This trial aims to determine whether the outcomes of trabeculotomy are noninferior to those of CTT in moderate PCG with a horizontal corneal diameter (HCD) of 12-14 mm.

Methods and analysis This is a 3-year, non-inferiority, prospective, randomised controlled trial. We plan to recruite 248 participants (aged $\leq 3$ years) with PCG with an HCD of 12-14 mm from the Department of Glaucoma, Zhongshan Ophthalmic Center, Guangzhou, China. One eye per participant will be randomly (1:1) assigned to receive trabeculotomy or CT. The primary outcome is the 3-year postoperative success rate in lowering intraocular pressure (IOP), and the secondary clinical outcomes will include IOP reduction, visual acuity, $\mathrm{HCD}$, central corneal thickness, axial length, cup-disc ratio, refractive error and postoperative complications. Data will be analysed by the intention-to-treat principle.

Ethical approval and dissemination The study protocol has been approved by the ethics committee of Zhongshan Ophthalmic Center (2014MEKY023) and the '5010 Plan' evaluation committee at Sun Yat-Sen University, Guangzhou, China. The results will be disseminated in international academic meetings and published in peer-reviewed journals. Trial registration number Chinese Clinical Trial Registry, ChiCTR-IOR-14005588; Date registered: 20 November 2014.

\section{INTRODUCTION}

Primary congenital glaucoma (PCG) is one of main causes of blindness in children. Liu et $a l^{1}$ reported that congenital glaucoma accounted for $5.1 \%$ of all congenital ocular diseases in a Chinese population. It is estimated to account for $0.01 \%-0.04 \%$ of blindness worldwide. ${ }^{2}$ In India, this disease accounts for $4.2 \%$ of all childhood blindness. ${ }^{2}$ Haddad $e t a \hat{l}$ evaluated 3210 visually impaired children and found that PCG was responsible for $10.2 \%$ of visual impairments. Since
Strengths and limitations of this study

- The trial design is prospective, randomised and controlled with a relatively large sample size, and the follow-up is comparatively long ( 3 years).

- The study is the first randomised controlled trial to comprehensively evaluate the surgical and visual outcomes of trabeculotomy and combined trabeculotomy-trabeculectomy in patients with primary congenital glaucoma with a horizontal corneal diameter (HCD) of 12-14 mm.

- This study assesses important clinical measurements with significant clinical implications, including HCD, axial length, central corneal thickness, cup-disc ratio, refractive errors and visual acuity. All data will be obtained following standardised protocols and assessed longitudinally.

- The intraocular pressure (IOP) criteria $(\leq 21 \mathrm{~mm} \mathrm{Hg})$ used to define glaucoma and surgical success rate may be relatively high for children younger than 3 years old.

Instead of the gold applanation tonometry, IOP is measured by Tono-Pen tonometry.

at least $50 \%$ of eyes with PCG presenting at birth will become legally blind, patients with PCG require prompt treatment and follow-up examinations throughout their lives. ${ }^{4}$

Surgical intervention is the main treatment for PCG. Goniotomy and trabeculotomy are considered initial procedures because of their high success rates. ${ }^{5}$ However, clear cornea is a premise for goniotomy but not necessarily for trabeculotomy. Trabeculotomy reduces intraocular pressure (IOP) by tearing the trabecular meshwork into the anterior chamber. Regarding complications, hyphema is more common in trabeculotomy but can resolve spontaneously and cause no additional problems. ${ }^{7-9}$

Combined trabeculotomy-trabeculectomy (CTT) has been advocated for treating 


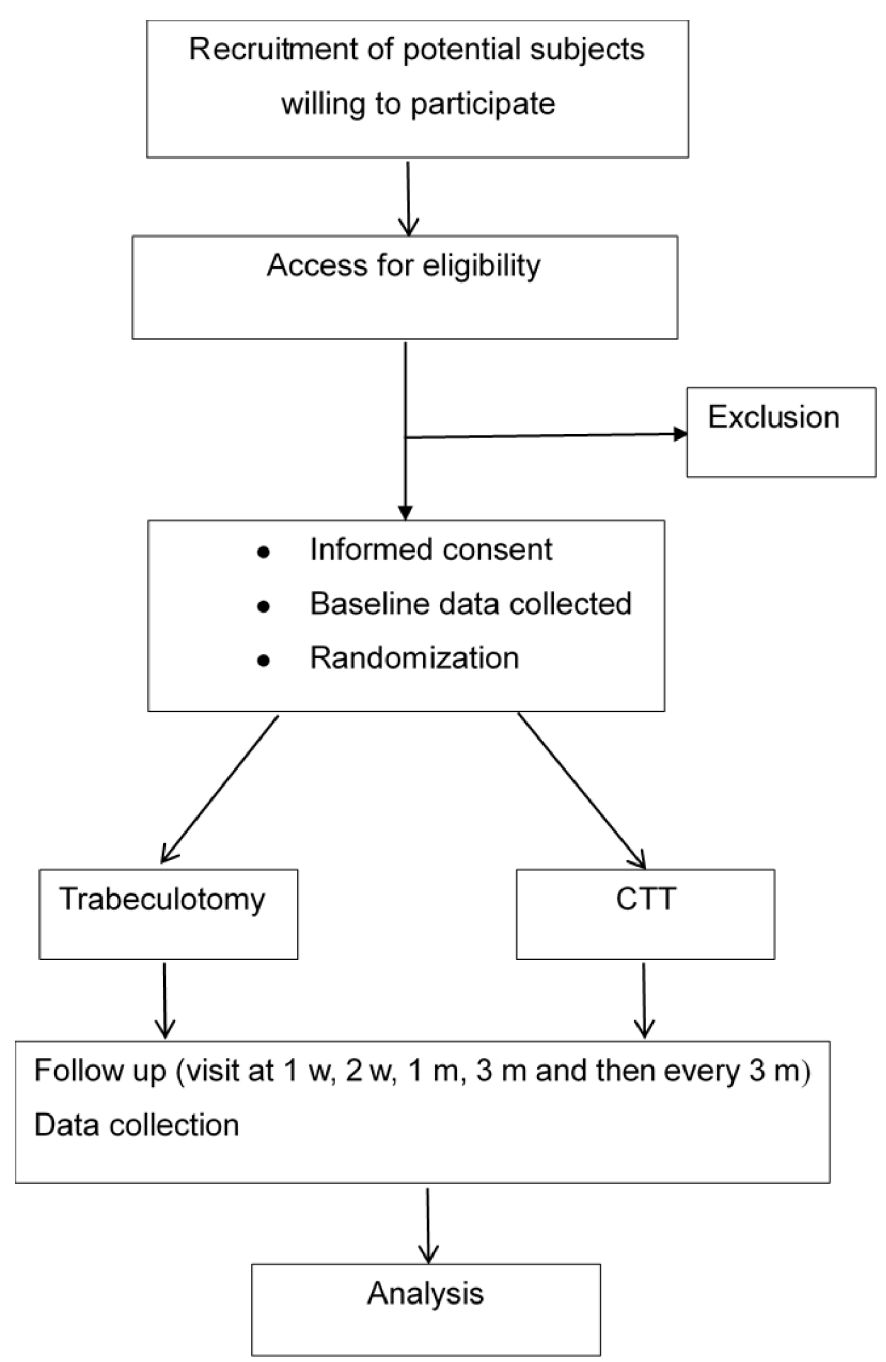

Figure 1 Flow chart of the study. CTT, combined trabeculotomy-trabeculectomy.

moderate-to-severe congenital glaucoma. ${ }^{10}$ The rationale for CTT is to gain access to the dual outflow through Schlemm's canal and the trabeculectomy fistula. The application of mitomycin-C (MMC) can improve the surgical success rates of CTT, ${ }^{11}$ which is, however, disputed by some other studies. ${ }^{12}{ }^{13}$ Complications after CTT surgery, such as hyphema, bleb-related infections, and choroidal detachment, have been reported. ${ }^{14}$

Although some studies have indicated that trabeculotomy and CTT are equally effective in lowering IOP, ${ }^{79} 15$ their results were inconsistent with others. ${ }^{10}{ }^{16}$ There is a paucity of randomised controlled trials with large sample sizes that compare the results of trabeculotomy with CTT for PCG. A randomised trial conducted by Khalil and Abdelhakim ${ }^{9}$ included a cohort of 28 eyes of 28 children younger than 2 years old with a mean follow-up time of 3 years. They concluded that both trabeculotomy and CTT with MMC had similar outcomes. However, due to limitations of sample sizes, it remains inconclusive as which procedure is preferable. ${ }^{17}$

The horizontal corneal diameter (HCD) is typically increased in patients with PCG, which serves as an indication for disease severity and a key factor for surgery selection. ${ }^{18}$ In general, angle surgeries are recommended for PCG with an HCD $<12 \mathrm{~mm}^{8}$ Trabeculectomy or CTT with or without the use of MMC is usually chosen for advanced cases with an HCD exceeding $14 \mathrm{~mm}^{7}{ }^{79}$ For moderate PCG with an HCD of 12-14 mm, trabeculotomy and CTT are the two major surgical options. However, it remains unknown whether trabeculotomy, when compared with CTT, yields comparable results and fewer postoperative complications in PCG with an HCD of 12-14 mm. Therefore, we design a study to determine whether the clinical outcomes of trabeculotomy are noninferior to those of CTT for PCG with an HCD of 12-14 $\mathrm{mm}$.

\section{Study objectives}

The primary outcome of our study is to compare the 3-year success rate in lowering IOP between trabeculotomy and CTT in patients with PCG with an HCD of 12-14 mm. The secondary outcome is to assess changes in IOP and the morphometric parameters of the eyeball, visual outcomes and postoperative complications in these two surgical procedures.

\section{METHODS AND ANALYSIS}

This protocol is developed in line with the Standard Protocol Items Recommendations for Interventional Trails (SPIRIT). The SPIRIT checklist for the protocol is available as online supplementary file. Protocol of this trial was approved on 25 August 2014.

\section{Trial design and setting}

This study is a 3-year, prospective, randomised, singlecentre, non-inferiority trial comparing clinical outcomes and postoperative complications between trabeculotomy and CTT in treating PCG with an HCD of 12-14 mm. Eligible patients will be enrolled and randomly assigned to receive either trabeculotomy or CTT (figure 1). The trial is being conducted at the Zhongshan Ophthalmic Center, Guangzhou, China.

\section{Participant selection}

\section{Inclusion criteria}

Participants will be included if they meet all of the following criteria: (1) diagnosis of PCG in either eye, (2) equal to or under 3 years of age, (3) HCD between 12 and $14 \mathrm{~mm}$ and (4) no previous intraocular surgery or laser treatment.

PCG is defined as follows ${ }^{20}$ :

1. Age $\leq 3$ years old.

2. IOP $>21 \mathrm{~mm} \mathrm{Hg}$.

3. Absence of other ocular or systemic diseases.

4. Combined with one or more of the following clinical signs: (1) corneal findings: Haab's striae, corneal oedema, corneal diameters $>11 \mathrm{~mm}$ in the newborns, $>12$ $\mathrm{mm}$ in children younger than 1 year old, and $>13 \mathrm{~mm}$ in children older than 1 year old; (2) increased $(>0.3)$ 
or asymmetric $(>0.2)$ cup-disc $(\mathrm{C} / \mathrm{D})$ ratio; and (3) abnormally increased axial length (AL).

Normal AL is as follows: 3 months-3 years: $19-22 \mathrm{~mm} .^{21}$

Only one eye per patient will be enrolled. If both eyes of a patient are eligible for the study, the eye with the higher baseline IOP will be selected. The treatment for the fellow eye will be determined at the physician's discretion.

\section{Exclusion criteria}

Patients will be excluded if they meet any of the following criteria:

- Inability of the patients' legal guardian to give informed consent.

- Inability of the patient to return to the clinic for the scheduled study visits.

- Contraindications to anaesthesia or surgery for ocular disease.

- Severe corneal cloudiness precluding anterior chamber visualisation.

- Secondary congenital glaucoma.

- Other coexisting ocular diseases such as an abnormal cornea, congenital iris abnormality, congenital cataract or retinopathy of prematurity.

\section{Withdrawal criteria}

1. Failure to locate or dissect Schlemm's canal by $120^{\circ}$.

2. The presence of any of the following issues during the operation: severe anaesthesia accident, suprachoroidal haemorrhage, or a change in the operative procedure according to the patient's condition.

3. A desire to quit the trial.

The withdrawal criteria described above have been established to ensure that the outcomes of the two procedures (trabeculotomy and CTT) will be effectively analysed for the full 3-year duration of the study.

\section{Interventions}

All surgeries will be performed under general anaesthesia by three attending surgeons (XL, MBY and MKL) who specialised in both types of surgery.

\section{Trabeculotomy}

This technique has been previously described. ${ }^{8}$ In brief, a superior quadrant fornix-based flap will be created. A 3 $\mathrm{mm} \times 3 \mathrm{~mm}$ superficial (12 o'clock) scleral flap of threequarters thickness will be made. A $2 \mathrm{~mm}$ radial incision will be made starting from the grey zone up to the white zone, followed by entering Schlemm's canal externally. An incision will be slowly deepened until the outer wall of Schlemm's canal is opened and seeping aqueous humour is observed. Schlemm's canal will be dissected by $120^{\circ}$ in both directions using a trabeculotome probe. The scleral flap will then be replaced with three interrupted 10-0 nylon sutures. The conjunctival flap will also be replaced with $8-0$ absorbable sutures.

Combined trabeculotomy-trabeculectomy

In the superior quadrant, CTT with MMC will be performed. A fornix-based conjunctival flap will be dissected. After dissection of a superficial (12 o'clock) scleral flap measuring $4 \times 3 \mathrm{~mm}^{2}$, MMC $(0.3 \mathrm{mg} / \mathrm{mL})$ soaked pieces of microsponge will be applied under the scleral flap and the conjunctiva for $3 \mathrm{~min}$, and the area will then be washed thoroughly with $30 \mathrm{~mL}$ of balanced salt solution. Then, trabeculotomy will be performed as described above. Trabeculectomy will be performed by cutting a $1 \mathrm{~mm} \times 2 \mathrm{~mm}$ deep scleral flap, followed by a peripheral iridectomy. The scleral flap and conjunctiva will then be replaced. Finally, the anterior chamber will be reformed with balanced salt solution.

Intraoperative data, including the duration of surgery, the same doses and duration of MMC used during the operation, anaesthesia accidents, intraoperative complications, such as hyphema, iris/vitreous damage, and trabeculotomyrelated problems, such as failure to identify Schlemm's canal or an inability to dissect Schlemm's canal by $120^{\circ}$, will be collected.

\section{Postoperative treatment and patient follow-up}

Patients will be treated with prednisolone acetate $1 \%$ (Allergan, Parsippany-Troy Hills, New Jersey, USA) six times daily in combination with topical antibiotics (tobramycin $0.3 \%$, s.a. ALCON-COUVREUR n.v) and pilocarpine 1\% (Bausch \& Lomb, Rochester, New York) four times daily for the first 4 weeks after the surgery.

Postoperative follow-up visits will be performed in the paediatric glaucoma clinic at week 1 , week 2 , week 4 , month 3 and then every 3 months ( \pm 1 weeks) for 3 years. The scheduled examinations of the follow-up visits are summarised in table 1 . Chloral hydrate $10 \%(0.8 \mathrm{ml} / \mathrm{kg}$, oral or rectal administration, the maximum dose is $10 \mathrm{~mL}$ per day) will be applied to patients not compliant for examinations.

If IOP is found to be high at a scheduled visit, topical antiglaucoma medication will be prescribed and the scheduled follow-up interval (if longer than 2 weeks) will be shortened to 2 weeks. Additional surgery will be performed if the IOP is $>21 \mathrm{~mm} \mathrm{Hg}$ on maximum antiglaucoma medications (including pilocarpine $1 \%$, brinzolamide $1 \%$ and latanoprost $0.005 \%$ ) in two consecutive study visits.

\section{Outcome assessment}

\section{Primary outcome}

The primary outcome is the success rate in lowering IOP at 3 years after surgery. Success is defined as:

1. IOP $\geq 5 \mathrm{~mm} \mathrm{Hg}$ and $\leq 21 \mathrm{~mm} \mathrm{Hg}$ on two consecutive follow-up visits with or without antiglaucoma medications. ${ }^{522}$

2. The absence of severe vision-threatening postoperative complications, such as suprachoroidal haemorrhage, retinal detachment or endophthalmitis.

3. No need for additional surgical intervention to control the IOP.

Complete success is defined as meeting success criteria without the need for antiglaucoma medications. Qualified success is defined as meeting success criteria with the use of antiglaucoma medications. 


\begin{tabular}{|c|c|c|c|c|c|c|c|c|c|c|c|c|c|c|c|c|c|}
\hline Visit number & 1 & 2 & 3 & 4 & 5 & 6 & 7 & 8 & 9 & 10 & 11 & 12 & 13 & 14 & 15 & 16 & 17 \\
\hline Examination & Baseline & Procedure & $\begin{array}{l}1 \mathrm{w} \\
\pm 2 \mathrm{~d}\end{array}$ & $\begin{array}{l}2 \mathrm{w} \\
\pm 2 \mathrm{~d}\end{array}$ & $\begin{array}{l}1 \mathrm{~m} \\
\pm 7 \mathrm{~d}\end{array}$ & $\begin{array}{l}3 \mathrm{~m} \\
\pm 7 \mathrm{~d}\end{array}$ & $\begin{array}{l}6 \mathrm{~m} \\
\pm 7 \mathrm{~d}\end{array}$ & $\begin{array}{l}9 \mathrm{~m} \\
\pm 7 \mathrm{~d}\end{array}$ & $\begin{array}{l}12 \mathrm{~m} \\
\pm 7 \mathrm{~d}\end{array}$ & $\begin{array}{l}15 \mathrm{~m} \\
\pm 7 \mathrm{~d}\end{array}$ & $\begin{array}{l}18 \mathrm{~m} \\
\pm 7 \mathrm{~d}\end{array}$ & $\begin{array}{l}21 \mathrm{~m} \\
\pm 7 \mathrm{~d}\end{array}$ & $\begin{array}{l}24 \mathrm{~m} \\
\pm 7 \mathrm{~d}\end{array}$ & $\begin{array}{l}27 \mathrm{~m} \\
\pm 7 \mathrm{~d}\end{array}$ & $\begin{array}{l}30 \mathrm{~m} \\
\pm 7 \mathrm{~d}\end{array}$ & $\begin{array}{l}33 \mathrm{~m} \\
\pm 7 \mathrm{~d}\end{array}$ & $\begin{array}{l}36 \mathrm{~m} \\
\pm 7 \mathrm{~d}\end{array}$ \\
\hline
\end{tabular}

\begin{tabular}{|c|c|c|c|c|c|c|c|c|c|c|c|c|c|c|c|c|c|}
\hline $\begin{array}{l}\text { Demographic } \\
\text { data }\end{array}$ & $x$ & & & & & & & & & & & & & & & & \\
\hline Medical history & $x$ & & & & & & & & & & & & & & & & \\
\hline $\begin{array}{l}\text { Physical } \\
\text { examination }\end{array}$ & $x$ & & & & & & & & & & & & & & & & \\
\hline IOP & $x$ & & $x$ & $x$ & $x$ & $x$ & $x$ & $x$ & $x$ & $x$ & $x$ & $x$ & $x$ & $x$ & $x$ & $x$ & $x$ \\
\hline $\mathrm{AL}$ & $x$ & & & & & $x$ & $x$ & & $x$ & & $x$ & & $x$ & & $x$ & & $x$ \\
\hline $\begin{array}{l}\text { Slit lamp } \\
\text { examination }\end{array}$ & $x$ & & $x$ & $x$ & $x$ & $x$ & $x$ & $x$ & $x$ & $x$ & $x$ & $x$ & $x$ & $x$ & $x$ & $x$ & $x$ \\
\hline $\begin{array}{l}\text { Fundus } \\
\text { photography* }\end{array}$ & $x$ & & & & & $x$ & $x$ & & $x$ & & $x$ & & $x$ & & $x$ & & $x$ \\
\hline $\begin{array}{l}\text { B-scan } \\
\text { ultrasound } \dagger\end{array}$ & $x$ & & & & & $x$ & $x$ & & $x$ & & $x$ & . & $x$ & & $x$ & & $x$ \\
\hline ССT & $x$ & & & & & & & & & & & & & & & & $x$ \\
\hline Medications & $x$ & & $x$ & $x$ & $x$ & $x$ & $x$ & $x$ & $x$ & $x$ & $x$ & $x$ & $x$ & $x$ & $x$ & $x$ & $x$ \\
\hline Adverse events & & $x$ & $x$ & $x$ & $x$ & $x$ & $x$ & $x$ & $x$ & $x$ & $x$ & $x$ & $x$ & $x$ & $x$ & $x$ & $x$ \\
\hline Reoperation & & & & & $x$ & $x$ & $x$ & $x$ & $x$ & $x$ & $x$ & $x$ & $x$ & $x$ & $x$ & $x$ & $x$ \\
\hline
\end{tabular}

In the event of non-visibility of the fundus,.

*Fundus photography and refraction will not be performed.

†B-scan ultrasound will be used to measure cupping.

AL, axial length; CCT, central corneal thickness; HCD, horizontal corneal diameter; IOP, intraocular pressure; VA, visual acuity.

\section{Secondary outcomes}

The secondary outcomes will be evaluated by IOP reduction and changes in the morphometric and functional parameters of eyeball: HCD, corneal transparency, CCT, C/D ratio, AL, VA and refraction. Postoperative complications, including hyphema, shallow anterior chamber, hypotony, surgery-related iridodialysis, complicated cataract, retinal or choroidal detachment, bleb complications (leakage or infection) and endophthalmitis, will be evaluated and recorded.

\section{Sample size calculation}

The sample calculation was based on the hypothesis that the 3-year success rate of trabeculotomy will be noninferior to that of CTT. Published studies have shown that the success rate at the third year after CTT ranges from $72.6 \%$ to $87 \%$. ${ }^{192324}$ We assume that the 3-year success rate of CTT will be approximately $80 \%$. Therefore, 224 subjects (112 per group) will be needed to provide the trial with a power of at least $80 \%$ to demonstrate the noninferiority ( $-15 \%$ margin) of trabeculotomy to CTT (onesided $\alpha$ value: 0.025 ). Assuming a $10 \%$ loss to follow-up, a sample size of 248 participants is required for this study, with 124 participants in each group.

\section{Patient recruitment and baseline data collection}

All subjects will first be assessed for potential participation in the study by the primary investigator. Patients who gave consent to the study will be invited to undergo enrolment examinations to determine enrolment status.

\section{Examinations}

IOP: IOP will be measured with a Tono-Pen Avia (Reichart, Depew, New York, USA) under sedation with chloral hydrate $10 \%$ and topical anaesthesia. Although the use of anaesthetic agents during examination under anaesthesia may influence IOP and affect the accuracy of IOP documentation, chloral hydrate has been shown to have a minimal effect on IOP in paediatric ophthalmic examinations. $^{18}$

Slit-lamp examination: the condition of the anterior segment, including corneal clarity, corneal oedema, Haab's striae, anterior chamber depth, iris, pupil and lens, will be evaluated using a hand-held slit lamp (Keeler, Bucks, England).

Corneal clarity will be recorded as mild (iris texture clearly seen), moderate (iris seen but texture not clearly visible) and severe (iris not visible). 
HCD: a calliper will be used to measure the HCD (white to white) by ophthalmologists. Participants with an HCD less than $12 \mathrm{~mm}$ or greater than $14 \mathrm{~mm}$ will be excluded.

CCT: CCT will be measured using ultrasound pachymetry (IOPac, Heidelberg Engineering, Heidelberg, Germany). Topical anaesthetic will be used prior to the application of the ultrasonic probe to the corneal surface. All measurements were taken with the child in the supine position. Ten measurements will be taken for each eye, and the lowest reading will be recorded.

$\mathrm{C} / \mathrm{D}$ ratio: the $\mathrm{C} / \mathrm{D}$ ratio will be evaluated using direct ophthalmoscopy (66 Vision, Suzhou, China) as permitted by the media clarity. Images showing the $\mathrm{C} / \mathrm{D}$ ratio will be obtained using a hand-held retinal camera (Kowanonmyd a-D III; KowaOptimedInc, Aichi, Japan) through a dilated pupil. For children with hazy media, whose fundus cannot be visualised, fundus photography will not be performed, and a B-scan ultrasound (Quantel Medical, CF, France) will be used to rule out any intraocular pathology and to detect excavation of the optic nerve head.

Ocular biometry: ocular biometry, including AL, anterior chamber depth, lens thickness and vitreous chamber depth, will be measured using A-scan ultrasound (Quantel Medical, CF, France). Ten repeated measurements will be taken and averaged for analysis.

Visual acuity (VA): VA will be measured using suitable procedures. Teller acuity cards (Vistech Consultants, Dayton, Ohio, USA) will be used at a distance of $55 \mathrm{~cm}$ in non-verbal children. The Lea symbols (Precision Vision, La Salle, Illinois, USA) with a test distance of $3 \mathrm{~m}$ and the Early Treatment of Diabetic Retinopathy Study LogMAR E chart (Precision Vision, Villa Park, Illinois, USA) with a test distance of $4 \mathrm{~m}$ will be employed for verbal children. Monocular VA will be assessed in the right eye followed by the left eye. For children who cannot complete the quantified VA examinations mentioned above, the ability to fix and follow light will be evaluated.

Refractive error: refraction will be measured by retinoscopy after cycloplegia. Cycloplegia will be induced with two drops of cyclopentolate $1 \%$ instilled 5 min apart, with a third drop administered after 20 min. Cycloplegia will be then evaluated after an additional $15 \mathrm{~min}$. Cycloplegia is considered complete if the pupil dilates to $\geq 6 \mathrm{~mm}$ and a light reflex is absent. ${ }^{25}$ Refractive error will not be evaluated when attempts to improve the view were not successful.

All ophthalmological examinations described above will be performed in both eyes. Chloral hydrate $10 \%$ will be applied to patients not compliant for examinations.

Written informed consent will be collected from each eligible participant's legal guardian prior to inclusion in the study. For eligible participants, demographic data (sex, date of birth and laterality), family history of PCG and medical history (age of onset, initial syndrome, age at diagnosis and medical treatment) will be recorded. Pregnancy and delivery information (gestational weeks, delivery mode, maternal drug intake and infection during pregnancy) will also be ascertained and recorded.

\section{Randomisation}

A randomisation list was generated with the SAS V.9.3 software package (SAS Institute) by a biostatistician who will not participate in data management. The 1:1 randomisation procedure will be performed using varying block sizes. To ensure concealment, the block size will not be disclosed. The allocation of patients will be concealed using sequentially numbered, opaque sealed envelopes. A total of 248 envelopes will be prepared by two researchers not involved in the study. For each recruited patient, his/ her group assignment will be revealed in the operating room on the day of surgery. Surgical management and intraoperative data will be collected.

Postoperative follow-up will be performed by investigators who will not participate in patient care and are trained to follow-up patients prior to the study. The surgeon(s) and investigator(s) will not communicate with each other while collecting data.

\section{Data management and monitoring}

All data collected at the scheduled follow-up visits (table 1) will be recorded in the case report forms and entered into a digital database by trained researchers. The soft copies of digital data will be stored in these devices and then in a server at the end of each visit day. The completed case report forms and hardcopy data forms will be kept in locked cabinets in the research centre. The implementation of the trial will be monitored by the principal investigator. Access to the final dataset will be limited to the trial administrator and the statistician.

\section{Statistical analysis}

All statistical analyses will be performed using SPSS V.22.0 (SPSS). Continuous variables conformed to the normal distribution will be expressed as the mean (SD). Dichotomous and nominal variables will be expressed as frequencies, ordinal and discrete variables as median and IQR.

The primary analysis will be based on the principle of intention to treat and will include all subjects who underwent randomisation, with data censored at the last schedule visit. We will perform a sensitivity analysis of the post hoc worst case scenario, in which subjects who did not complete follow-up were considered failed outcomes, and a sensitivity analysis of a post hoc complete-case scenario, in which only subjects who had complete data all through the trail will be included. We calculated $95 \%$ CI for the estimates of the absolute differences between the two treatment groups regarding the 3-year success rate using the Cochran-Mantel-Haenszel method. Noninferiority would be met if the lower limit of the $95 \%$ CI of the absolute difference did not cross the prespecified non-inferiority margin $(-15 \%)$. The survival data (timeto-IOP controlled) will be analysed using the KaplanMeier method. The log-rank test will be employed to compare curves in the trabeculotomy and CTT groups.

Secondary outcomes will be assessed with two-sided tests. Comparisons of continuous variables distributed normally, such as the IOP, HCD, AL and central corneal 
thickness (CCT) will be performed between the two groups using Student's t-test. Considering that the normal IOP in children is lower than that of adults, we will use 18 $\mathrm{mm} \mathrm{Hg}$ as a cut-off and compare the surgical outcomes between two groups after 3 years of follow-up using the Kaplan-Meier method. For continuous variables not distributed normally and for discrete variables (including the $\mathrm{C} / \mathrm{D}$ ratio, number of antiglaucoma drugs and distribution of refractive errors) between the two groups comparison will be performed using Mann-Whitney U test. The Chi-square test or Fisher's exact test will be used to compare the proportions of the visual outcomes, and complications between the two surgical groups.

\section{Safety consideration}

The safety evaluations of the study will include complications associated with surgeries as well as drugs adverse events. The procedures and drugs used in the study are routinely administered in daily practice. Thus, the trial has risks not exceeding usual clinical care that the patients would otherwise receive. Throughout the study, all adverse events will be recorded and managed.

Drug-related complications include unanticipated events caused by cycloplegia, antiglaucoma drugs and chloral hydrate. Dilation will be established following a slit lamp examination. Doctors will closely monitor the patients' pupil reflexes and vital signs after administering the medications.

Vision-threatening complications, such as suprachoroidal haemorrhage, retinal detachment and endophthalmitis, will constitute major adverse events.

\section{Trial status}

Recruitment began in the first quarter of 2015. Currently, $75 \%$ of the sample size has been attained. It is anticipated that the study will reach the recruitment target of 248 participants by the fourth quarter of 2019. There are no plans for interim analysis.

\section{Patient and public involvement}

Patients and public were not involved in the design of the study.

\section{ETHICS AND DISSEMINATION}

The study results will be presented at national and international meetings on ophthalmology.

\section{DISCUSSION}

This is a prospective, randomised, controlled intervention trial that aims to provide evidence for clinicians for better judgement regarding surgical options for patients with PCG. To the best of our knowledge, this trial is the largest clinical trial in the field of paediatric glaucoma. The findings are expected to provide evidence indicating whether trabeculotomy is non-inferior to CTT in treating PCG with an HCD of 12-14 mm.

For PCG with an HCD less than $12 \mathrm{~mm}$, the anatomic abnormality of Schlemm's canal is usually not significant, facilitating its identification during the operation. As a result, angle surgery alone is sufficient to lower IOP in these patients. Sampaolesi $e t a t^{26}$ proposed that trabeculotomy is suitable for children with PCG in whom the corneal diameter is less than $13 \mathrm{~mm}$ and the AL is less than $23 \mathrm{~mm}$. Advanced PCG with an HCD greater than $14 \mathrm{~mm}$ is usually associated with a significant anatomic anomaly of the anterior drainage angle. The abnormally stretched anatomy of the limbus in these patients frequently makes it difficult to clearly identify the lumen of Schlemm's canal that has to be cannulated for the trabeculotomy. Thus, the success rate of trabeculotomy is lower in advanced PCG cases. Quigley ${ }^{27}$ reported the results of trabeculotomy in 28 eyes with congenital glaucoma. The success rate in eyes with an HCD greater than $14 \mathrm{~mm}$ was $67 \%$ compared with $100 \%$ in eyes with a smaller HCD. Both of the conditions described above will lead to biases in the results. Moreover, most PCG cases in China have an HCD ranging from $12 \mathrm{~mm}$ to $14 \mathrm{~mm},{ }^{728}$ and these patients have a good chance of preserving useful VA if treated correctly.

No unified guideline is currently available to determine PCG severity based on corneal diameter. Cronemberger et $a l^{29}$ confirmed that a higher HCD will trigger higher HCD and AL at final follow-up. Kiskis $e t a l^{30}$ studied the HCD and AL in patients with PCG and concluded that $\mathrm{HCD}$ measurement was a more reliable guide than AL in the assessment of PCG. Currently, we are unaware of any studies that compared long-term outcomes between CTT and trabeculotomy in patients with PCG who exhibited homogeneity in terms of disease severity. After considering the above information, we selected an HCD of $12-14 \mathrm{~mm}$ as an inclusion criterion. However, selection of surgical methods for the treatment of PCG and the evaluation of PCG severity based on HCD alone are issues requiring further investigation and improvement.

With regard to IOP, we selected an IOP value of $\leq 21$ $\mathrm{mm} \mathrm{Hg}$ as a success criterion based on the previous reports. ${ }^{53} 31$ In this study, IOP will be measured with Tono-Pen which has been widely used in clinic for many years. We chose Tono-Pen as the measurement by referring to the previous studies. ${ }^{32-34}$ On the other hand, Tono-Pen is particularly useful with corneal scars or oedema, ${ }^{35}$ which are often seen in PCG eyes. We used Tono-Pen for all patients at each scheduled visit, which eliminated any possibility of bias due to the use of different tonometry techniques in different patients.

In conclusion, this is a large clinical trial aiming to provide evidence for the optimum first-line surgery for patients with PCG with an HCD of 12-14 mm. If the trabeculotomy group is associated with comparable surgical success and fewer postoperative complications compared with CTT group, trabeculotomy should be recommended as a primary surgical treatment for PCG with an HCD of 12-14 mm, saving trabeculectomy for future intervention. In addition, complications associated with trabeculectomy will be reduced. The visual outcome in this trial may help provide insight into the effects of surgical methods on VA. The findings of our study are expected to provide guidance 
to clinicians weighing the benefit and risk of trabeculotomy compared with CTT for the treatment of PCG.

Acknowledgements The authors would like to thank all of the research assistants and nursing staff involved in this trial for contributing to the practical organisation and execution of this study.

Contributors $\mathrm{XL}$ conceived the study and is the project leader for the trial. $\mathrm{XL}, \mathrm{XG}$, $Y Y, J H, Y Z, X C, J H$ and LF participated in the study design and recruited the patients. LF wrote the manuscript. XL, XG, YZ and XX critically revised the manuscript. JZ designed the database system and performed the statistics-related design. All authors read and approved the final manuscript.

Funding This study was supported by the Sun Yat-Sen University Clinical Research 5010 Program (2014016) and the National Natural Science Foundation of China (81800879)

Competing interests None declared.

Patient consent for publication Not required.

Ethics approval This study was approved by the ethics committee of Zhongshan Ophthalmic Center (reference number 2014MEKY023). The study protocol was also reviewed by the "5010 Plan" evaluation committee at Sun Yat-sen University, Guangzhou, China. Every year, the evaluation committee will examine the study progress and its adherence to the study protocol. Any important modifications to the protocol will be documented in the study protocol as formal amendments. These amendments will be submitted to the ethics committee of Zhongshan Ophthalmic Center and the "5010 Plan" evaluation committee of the Sun Yat-sen University for a review. The project leader will ensure that this study is conducted in accordance with the principles of the World Medical Association Declaration of Helsinki.

Provenance and peer review Not commissioned; externally peer reviewed.

Open access This is an open access article distributed in accordance with the Creative Commons Attribution Non Commercial (CC BY-NC 4.0) license, which permits others to distribute, remix, adapt, build upon this work non-commercially, and license their derivative works on different terms, provided the original work is properly cited, appropriate credit is given, any changes made indicated, and the use is non-commercial. See: http://creativecommons.org/licenses/by-nc/4.0/.

\section{ORCID iD}

Xing Liu http://orcid.org/0000-0003-4840-9887

\section{REFERENCES}

1 Liu B, Huang W, He M, et al. [An investigation on the causes of blindness and low vision of students in blind school in Guangzhou]. Yan Ke Xue Bao 2007;23:117-20.

2 Mandal AK, Chakrabarti D. Update on congenital glaucoma. Indian J Ophthalmol 2011;59 Suppl:148-57.

3 Haddad MAO, Sei M, Sampaio MW, et al. Causes of visual impairment in children: a study of 3,210 cases. J Pediatr Ophthalmol Strabismus 2007:44:232-40.

4 Levy J, Tessler Z, Tamir O, et al. [Primary congenital glaucoma]. Harefuah 2004;143:876-80.

5 Bowman RJC, Dickerson M, Mwende J, et al. Outcomes of goniotomy for primary congenital glaucoma in East Africa. Ophthalmology 2011;118:236-40.

6 Morales J, Al Shahwan S, Al Odhayb S, et al. Current surgical options for the management of pediatric glaucoma. J Ophthalmol 2013;2013:1-16.

7 Zhang X, Du S, Fan Q, et al. Long-Term surgical outcomes of primary congenital glaucoma in China. Clinics 2009;64:543-51.

8 Huang J-L, Huang J-J, Zhong Y-M, et al. Surgical outcomes of trabeculotomy in newborns with primary congenital glaucoma. Chin Med J 2016;129:2178-83.

9 Khalil DH, Abdelhakim MASE. Primary trabeculotomy compared to combined trabeculectomy-trabeculotomy in congenital glaucoma: 3-year study. Acta Ophthalmol 2016;94:e550-4.

10 Al-Hazmi A, Awad A, Zwaan J, et al. Correlation between surgical success rate and severity of congenital glaucoma. $\mathrm{Br} J$ Ophthalmol 2005;89:449-53.
11 Hsu C-R, Chen Y-H, Tai M-C, et al. Combined trabeculotomytrabeculectomy using the modified safer surgery system augmented with MMC: its long-term outcomes of glaucoma treatment in Asian children. Graefes Arch Clin Exp Ophthalmol 2018;256:1187-94.

12 Rodrigues AM, Júnior AP, Montezano FT, et al. Comparison between results of trabeculectomy in primary congenital glaucoma with and without the use of mitomycin C. J Glaucoma 2004;13:228-32.

13 Ozkiris A, Tamcelik N. Long-Term results of trabeculectomy with different concentrations of mitomycin $\mathrm{C}$ in refractory developmental glaucoma. J Pediatr Ophthalmol Strabismus 2005;42:97-102.

14 Jalil A, Au L, Khan I, et al. Combined trabeculotomy-trabeculectomy augmented with 5-fluorouracil in paediatric glaucoma. Clin Exp Ophthalmol 2011;39:207-14.

15 Biedner BZ, Rothkoff L. Combined trabeculotomy-trabeculectomy compared with primary trabeculotomy for congenital glaucoma. $J$ Pediatr Ophthalmol Strabismus 1998;35:49-50.

16 Chen TC, Chen PP, Francis BA, et al. Pediatric glaucoma surgery: a report by the American Academy of ophthalmology. Ophthalmology 2014;121:2107-15.

17 Ghate D, Wang X. Surgical interventions for primary congenital glaucoma. Cochrane Database Syst Rev 2015;1:CD008213.

18 Yu Chan JY, Choy BN, Ng AL, et al. Review on the management of primary congenital glaucoma. J Curr Glaucoma Pract 2015;9:92-9.

19 Mandal AK, Matalia JH, Nutheti R, et al. Combined trabeculotomy and trabeculectomy in advanced primary developmental glaucoma with corneal diameter of $14 \mathrm{~mm}$ or more. Eye 2006;20:135-43.

20 Thau A, Lloyd M, Freedman S, et al. New classification system for pediatric glaucoma: implications for clinical care and a research registry. Curr Opin Ophthalmol 2018;29:385-94.

21 Bach A, Villegas VM, Gold AS, et al. Axial length development in children. Int J Ophthalmol 2019;12:815-9.

22 Lawrence SD, Netland PA. Trabeculectomy versus combined trabeculotomy-trabeculectomy in pediatric glaucoma. $J$ Pediatr Ophthalmol Strabismus 2012;49:359-65.

23 Mandal AK, Bhatia PG, Bhaskar A, et al. Long-Term surgical and visual outcomes in Indian children with developmental glaucoma operated on within 6 months of birth. Ophthalmology 2004;111:283-90.

24 Mandal AK, Gothwal VK, Nutheti R. Surgical outcome of primary developmental glaucoma: a single surgeon's long-term experience from a tertiary eye care centre in India. Eye 2007;21:764-74.

25 Negrel AD, Maul E, Pokharel GP, et al. Refractive error study in children: sampling and measurement methods for a multi-country survey. Am J Ophthalmol 2000;129:421-6.

26 Sampaolesi R, Zarate J, Sampaolesi JR. The glaucomas. V. I. pediatric glaucomas. Leipzig, Germany: Springer-Verlag Berlin Heidelberg, 2009.

27 Quigley HA. Childhood glaucoma: results with trabeculotomy and study of reversible cupping. Ophthalmology 1982;89:219-26.

28 Cai $Y$, Li M-Y, Shen Y-Y, et al. [Long-term effect of trabeculotomy on primary congenital glaucoma]. Zhonghua Yan Ke Za Zhi 2004;40:733-6.

29 Cronemberger S, Calixto N, Avellar Milhomens TG, et al. Effect of intraocular pressure control on central corneal thickness, horizontal corneal diameter, and axial length in primary congenital glaucoma. $J$ Aapos 2014:18:433-6.

30 Kiskis AA, Markowitz SN, Morin JD. Corneal diameter and axial length in congenital glaucoma. Can J Ophthalmol 1985;20:93-7.

31 Essuman VA, Braimah IZ, Ndanu TA, et al. Combined trabeculotomy and trabeculectomy: outcome for primary congenital glaucoma in a West African population. Eye 2011;25:77-83.

32 Ben-Zion I, Tomkins O, Moore DB, et al. Surgical results in the management of advanced primary congenital glaucoma in a rural pediatric population. Ophthalmology 2011;118:231-5.

33 Yalvac IS, Satana B, Suveren A, et al. Success of trabeculotomy in patients with congenital glaucoma operated on within 3 months of birth. Eye 2007;21:459-64.

34 Sahin A, Tüfek A, Cingü AK, et al. The effect of I-gel TM airway on intraocular pressure in pediatric patients who received sevoflurane or desflurane during strabismus surgery. Paediatr Anaesth 2012;22:772-5.

35 Yilmaz I, Altan C, Aygit ED, et al. Comparison of three methods of tonometry in normal subjects: Goldmann applanation tonometer, non-contact airpuff tonometer, and Tono-Pen XL. Clin Ophthalmol 2014;8:1069. 